\title{
Intelligent Hybrid Cheapest Cost and Mobility Optimization RAT Selection Approaches for Heterogeneous Wireless Networks
}

\author{
Abdallah AL Sabbagh, Robin Braun, and Mehran Abolhasan \\ Centre for Real-Time Information Networks (CRIN), University of Technology, Sydney (UTS), Sydney, Australia \\ E-mail: \{abdallah.alsabbagh, robin.braun, mehran.abolhasan\}@uts.edu.au
}

\begin{abstract}
The evolution of wireless networks has led to the deployment of different Radio Access Technologies (RATs) such as UMTS Terrestrial Radio Access Network (UTRAN), Long Term Evolution (LTE), Wireless Local Area Network (WLAN) and Mobile Worldwide Interoperability for Microwave Access (WiMAX) which are integrated through a common platform. Common Radio Resource Management (CRRM) was proposed to manage radio resource utilization in heterogeneous wireless networks and to provide the required Quality of Service $(\mathrm{QoS})$ for allocated calls. RAT selection algorithms are an integral part of the CRRM algorithms. Their role is to decide, when a new or Vertical Handover (VHO) call is requested, which of the available RATs is most suitable to fit the need of the incoming call and when to admit them. This paper extends our earlier work on the proposed intelligent mobility optimization and proposes an intelligent hybrid cheapest cost RAT selection approach which aims to increase users' satisfaction by allocation users that are looking for cheapest cost connections to a RAT that offers the cheapest cost of service. A comparison for the performance of centralized loadbalancing, proposed and distributed cheapest cost and mobility optimization algorithms is presented. Simulation results show that the proposed intelligent algorithms perform better than the centralized load-balancing and the distributed algorithms.
\end{abstract}

Index Terms-Next Generation Wireless Network (NGWN); Heterogeneous Wireless Network; Common Radio Resource Management (CRRM); Radio Access Technology (RAT) Selection Algorithms; Mobility Optimization algorithm; Cheapest Cost algorithm

\section{INTRODUCTION}

Due to the increasing demand of wireless services, mobile technology has rapidly progressed towards the fourth generation $(4 \mathrm{G})$ networking paradigm, which will provide high speed network connections for a variety of services such as Voice over IP (VoIP), web browsing, File Transfer Protocol (FTP), video streaming, online gaming, real time video and push-to-talk [1] and will interconnect different Radio Access Technology (RATs)

This article is an extended version of the paper titled: "A Mobility Optimization CRRM Approach for Next Generation Wireless Networks" by A. AL Sabbagh, R. Braun and M. Abolhasan which appeared in the International Conference on Computer \& Information Sciences (ICCIS 2012), Kuala Lumpur, Malaysia, June 12-14, 2012. such as: UMTS Terrestrial Radio Access Network (UTRAN), Long Term Evolution (LTE), Wireless Local Area Network (WLAN) and Mobile Worldwide Interoperability for Microwave Access (WiMAX) through a common platform [2-4].

Currently, Radio Resource Management (RRM) including admission control, congestion control, power control, packet scheduling, horizontal handover (HO), initial RAT selection algorithm and vertical HO (VHO) algorithms are implemented efficiently for the specific RAT it was developed for. However, they are not suitable for heterogeneous wireless networking scenarios where various types of RATs exist. Common Radio Resource Management (CRRM) was proposed to manage radio resource utilization in heterogeneous wireless networks [5].

A number of different RAT selection algorithms have been proposed in the literature for heterogeneous wireless networks. A terminal modality based RAT selection algorithm which aims to reduce the call blocking and dropping probability, and to guarantee fairness radio resources allocation among the heterogeneous wireless networks is proposed in [6]. The proposed algorithm makes RAT selection decisions based on mobile terminal capability, network load, and terminal support index of each of the available RAT. A network based RAT selection algorithm is proposed in [7]. This algorithm aims to optimize the radio resource utilization while ensuring acceptable Quality of Service (QoS) provision to the end users. Qiang et al. proposed a VHO RAT selection mechanism for multimedia traffic in a multiRAT scenario which includes WLAN and Code Division Multiple Access (CDMA) RATs using a link utility function [8]. The proposed algorithm aims to guarantee the user's QoS requirements and to minimize the congestion, balance the traffic load and maximize the overall network reward.

The proposed RAT selection algorithms can be categorized into centralized such as load-balancing algorithm and policy based algorithm or distributed algorithms such as service based algorithm $[9,10]$.

Centralized RAT selection algorithms have the benefit of considering more criteria during the making decision process. However, centralized algorithms do not guarantee required QoS for all admitted calls. In addition, 
they reduce network capacity as a result of the introduced signaling overheads or delay resulted by the communication between the network entities.

On the other hand, distributed algorithms have the benefit of considering users' preferences. A number of different distributed algorithms are proposed in [11-13]. These algorithms allow UT to select the most efficient RAT that maximizes its satisfaction which is based on its preference such as best QoS or cheapest cost. However, distributed algorithms do not take into account the network benefits and policies. This may lead to inefficient radio resource utilization and it may create network bottlenecks.

All the proposed algorithms have limitations and do not provide a complete solution for the RAT selection problem. Therefore there is a need for a hybrid RAT selection algorithm (distributed with network assistance) which will provide the UTs some information that assists them during their decision making process to select the most efficient RAT that maximizes their satisfactions and at the same time improve the efficient radio resource utilization. Previously, we have proposed an intelligent mobility optimization RAT selection approach in [14] and an intelligent power efficient RAT selection approach in [15]. In this paper, we extend our earlier work on the proposed intelligent mobility optimization approach in [14] and propose an intelligent hybrid cheapest cost RAT selection approach [16] which also uses the IEEE P1900.4 Protocol [17] to enable communication between the UTs and the different wireless networks. The proposed approach also aims to improve the RRM in heterogeneous wireless networks. This improvement will lead to enhanced QoS for end user, increase users' satisfactions and improve the efficiently of radio resource utilization. The proposed intelligent cheapest cost RAT selection approach is then compared with the proposed mobility optimization approach against centralized loadbalancing and distributed algorithms.

The remainder of the paper is organized as follows. Section II presents the proposed intelligent mobility optimization algorithm and proposes an intelligent cheapest cost algorithm. In Section III, the cost functions for the allocation of radio resources for both intelligent mobility optimization and cheapest cost algorithms are presented. The simulation environment and parameters where the proposed intelligent mobility optimization and intelligent cheapest cost algorithms are compared against the centralized load-balancing and the distributed mobility optimization and cheapest cost algorithms is presented In Section IV. The five algorithms are simulated in a scenario which UTRAN, LTE and WLAN are overlapped in the same geographical area. A comparison for the performance of centralized loadbalancing, distributed and proposed mobility optimization and cheapest cost algorithms is presented in Section V. These algorithms are evaluated in terms of new call blocking probability, VHO call dropping probability, users' satisfactions probability, average satisfaction increasing percentages, load utilization, average cell throughput, average cost per call and average saved cost percentage per call. Finally, the paper is concluded in Section VI.

\section{PROPOSED INTELLIGENT HybRID RAT SELECTION APPROACHES}

Centralized RAT selection algorithms do not guarantee the required QoS for the admitted calls and reduce the network capacity. Distributed RAT selection algorithms do not consider network preferences and policies. In this section, we present the intelligent hybrid RAT selection approach for mobility optimization [14] and propose an intelligent hybrid cheapest cost RAT selection approach which uses the IEEE P1900.4 Protocol [17] to enable communication between the UTs and the different wireless networks. More details on each of these approaches are shown in the following subsections.

\section{A. Intelligent Mobility Optimization Algorithm}

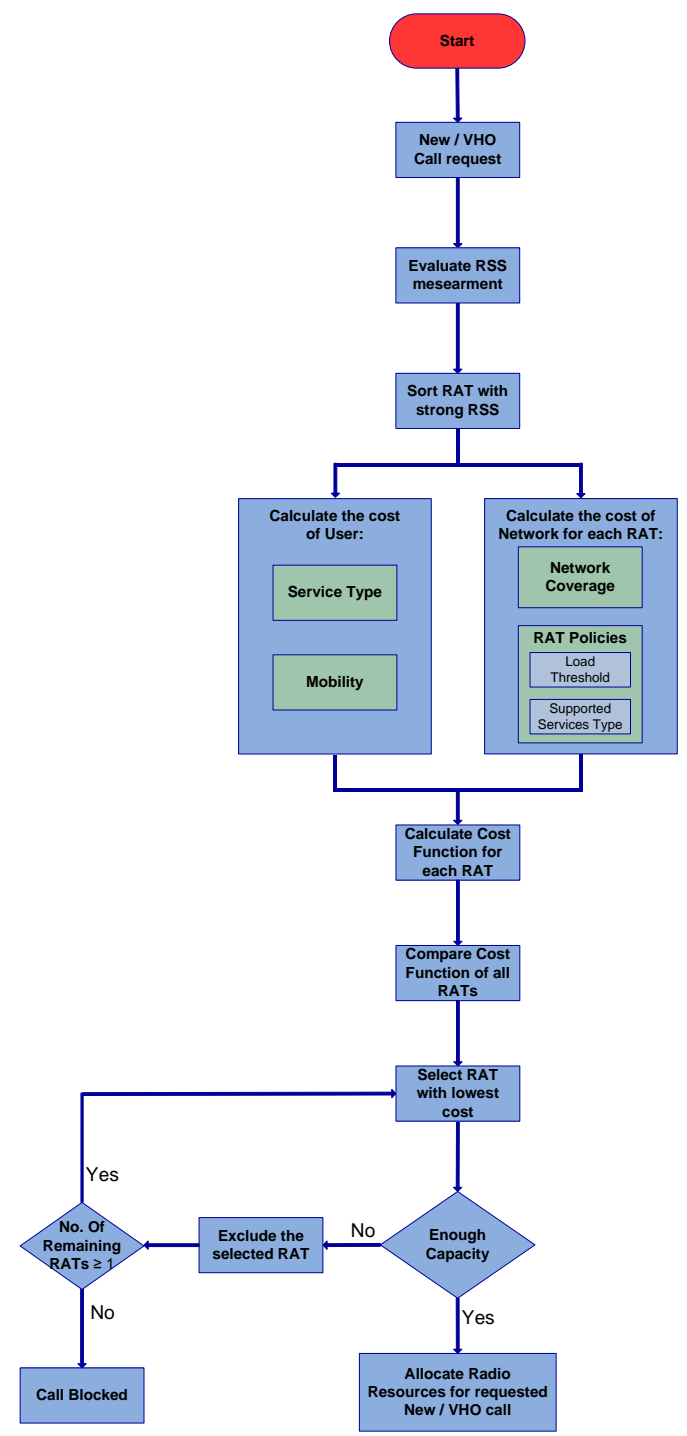

Figure 1. A mobility optimization RAT selection approach

Different users can be in different velocity types such as low, medium or high mobility. The mobility optimization algorithm aims to reduce unnecessary handover. Therefore, low mobility UTs can be allocated to a RAT that has small coverage area such as WLAN; 
however, high mobility UTs can be allocated to a RAT that has higher coverage area such as WWAN technologies. In addition, RATs policies which include supported services types and load threshold policies need to be considered.

The proposed intelligent hybrid RAT selection approach for mobility optimization will consider the above factors for each new or VHO call and allocate each call to an optimal RAT depending on the UT requirements and the current RATs status.

The flow chart of the proposed intelligent hybrid RAT selection approach for mobility optimization is presented in Fig. 1. When a new or VHO call is requested, UT will first evaluate the Received Signal Strength (RSS) measurements and makes a list of available RATs that have a strong RSS to accommodate the requested call. Then, the UT will collect information on each RAT using the IEEE P1900.4 Protocol. These information will cover network coverage costs of each RAT and RAT policy attributes such as supported services type and load threshold. A cost will be given for each attribute for each RAT. Other costs will be given for the user attributes, such as: requested service type, user mobility status (low, medium or high). After that, a cost function for each RAT is calculated. Then, a comparison between these costs is made. The RAT with the lowest cost will be allocated for the requested call. If the selected RAT does not have enough capacity to allocate the requested call, another RAT will be selected. If none of the available RATs have enough capacity to serve the requested call, the call will be dropped or blocked.

\section{B. Intelligent Cheapest Cost Algorithm}

Users may prefer to be connected to a RAT that offers cheapest cost of services in their relaxation time. Each RAT has a different service cost compared to others. Some RATs may offer cheaper cost than other RATs for all service types, as an example: WLAN has cheapest cost than WWAN technologies. Other RATs may offer cheaper cost for one service type and may be more expensive for other service types. Therefore, users preferring cheapest cost of services can be allocated to the RAT that offers the cheapest cost for the requested services. These allocations are not always the optimal selections, especially when the UTs are in high mobility. Allocation users looking for cheapest cost to WLAN are not the best choice for this case, where unnecessary handovers will be increasing. Another factor is the RATs policies, where each RAT may support different service types and may have different policies against the load threshold.

In the intelligent cheapest cost algorithm, the smart device will collect information on each RAT using the IEEE P1900.4 Protocol. This information will cover the service cost (low, medium or high) of each RAT for the requested call and RAT policies attributes such as supported services type and load threshold for new and VHO calls as shown in Fig. 2. A cost will be given for each attribute for each RAT. Other costs will be given for the user attributes (requested service type and user mobility status). After that, the cost function of radio resources allocation for each RAT will be calculated. The RAT with the lowest cost of allocation will be selected for the requested call. If the selected RAT does not have enough capacity to allocate the requested call, another RAT will be selected. If none of the available RATs have enough capacity to serve the requested call, the call will be dropped or blocked.

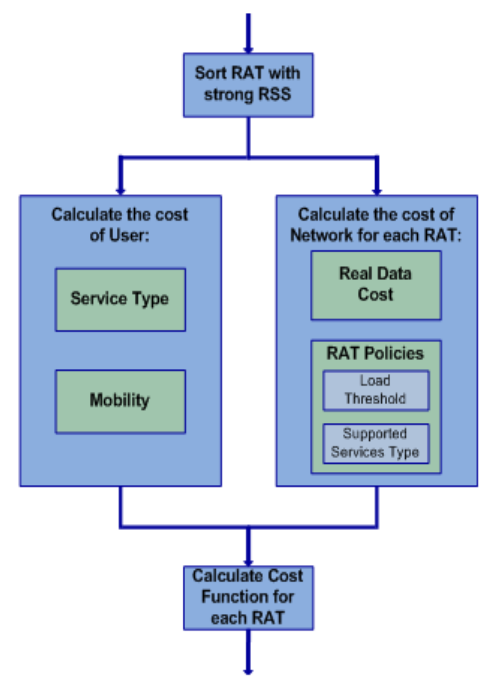

Figure 2. Cost function factors for the cheapest cost RAT selection approach.

\section{COST FuUnction MOdEl FOR THE PROPOSED ALGORITHMS}

This section presents the cost function for the allocation of radio resources in the proposed intelligent mobility optimization and the proposed intelligent cheapest cost RAT selection approaches for the requested user calls in heterogeneous wireless networks. Calls will be allocated to the RAT that has the lowest cost of allocation. If the selected RAT is unable to serve a call, another RAT will be selected. If none of the RATs are able to serve the call, it will be blocked/dropped. The next subsections calculate the cost of allocation for each of the proposed intelligent RAT selection approaches.

\section{A. Intelligent Mobility Optimization Algorithm}

The cost of allocation for the proposed intelligent mobility optimization approach is calculated by the following equation:

$$
C=C_{N}+C_{U}
$$

where

$\mathrm{C}_{\mathrm{N}}$ is the cost of network.

$\mathrm{C}_{\mathrm{U}}$ is the cost of user.

The cost of network is calculated as follows:

$$
C_{N}=\frac{\sum_{j=1}^{N}\left(W_{j} \times P_{j}\right)}{\sum_{j=1}^{N} W_{j}}
$$

where 
$P_{j}$ is the parameter related to allocation of resources in the network, such as: network coverage cost $\left(\mathrm{P}_{\mathrm{NC}}\right)$, supported service type cost $\left(\mathrm{P}_{\mathrm{SST}}\right)$ and load threshold cost $\left(\mathrm{P}_{\mathrm{LTH}}\right)$. cost.

$\mathrm{W}_{\mathrm{j}}$ is the factor weight for each network parameter

By adding the above parameters and weight factors, Equation (2) will be:

$$
C_{N}=\frac{W_{N C} \times P_{N C}+W_{S S T} \times P_{S S T}+W_{L T H} \times P_{L T H}}{W_{N C}+W_{S S T}+W_{L T H}}
$$

The cost of user is calculated as follows:

$$
C_{U}=\frac{\sum_{i=1}^{N}\left(W_{i} \times P_{i}\right)}{\sum_{i=1}^{N} W_{i}}
$$

where

$P_{i}$ is the parameter related to the usage of resources depending on user status, such as: requested service type cost $\left(\mathrm{P}_{\mathrm{RST}}\right)$ and cost of mobility $\left(\mathrm{P}_{\mathrm{M}}\right)$.

$\mathrm{W}_{\mathrm{i}}$ is the factor weight for each user parameter cost.

By adding the above parameters and weight factors, Equation (4) will be:

$$
C_{U}=\frac{W_{R S T} \times P_{R S T}+W_{M} \times P_{M}}{W_{R S T}+W_{M}}
$$

\section{B. Intelligent Cheapest Cost Algorithm}

In the proposed intelligent cheapest cost algorithm, the cost function for the allocation of radio resources of each RAT is calculated by using Equation (1).

The cost of network for the cheapest cost algorithm is calculated by using Equation (2), where:

$P_{j}$ is the parameter related to allocation of resources in the network, such as: cost of real data cost $\left(\mathrm{P}_{\mathrm{RDC}}\right)$, supported service type cost $\left(\mathrm{P}_{\mathrm{SST}}\right)$ and load threshold cost $\left(\mathrm{P}_{\mathrm{LTH}}\right)$. cost.

$\mathrm{W}_{\mathrm{j}}$ is the factor weight for each network parameter

By adding the above parameters and weight factors, Equation (2) will be:

$$
C_{N}=\frac{W_{R D C} \times P_{R D C}+W_{S S T} \times P_{S S T}+W_{L T H} \times P_{L T H}}{W_{R D C}+W_{S S T}+W_{L T H}}
$$

The cost of user for the cheapest cost algorithm is calculated by using Equation (5).

\section{Simulation ENVIRONMENT AND PARAMETERS}

The comparison for the performance of centralized load-balancing, distributed and the proposed mobility optimization and cheapest cost algorithms is simulated in a scenario which is assumed that UTRAN, LTE and WLAN are overlapped in the same geographical area. The area which has coverage for the three RATs is named: hotspot area. The network topology is shown in Fig. 3.
It is assumed that users arrive and can move inside or outside the hotspot area. Three different service types are considered: voice, data and video calls. Two different video call types are considered: video calls with low resolution and video calls with high resolution (video conference calls). It is assumed that the calls arrivals are generated according to a Poisson process with a mean rate of $\lambda$ [18]. It is also assumed that each call is held for an exponentially distributed call duration time with a mean of $1 / \mu$ [18].

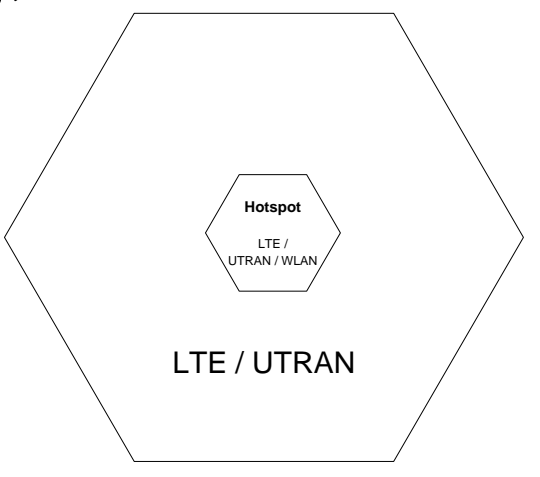

Figure 3. Multi-access cellular network

In UTRAN, the transmission scheme is based on WCDMA. Voice calls are accepted with a data rate of 12.2 kbps. Video calls with low resolution are accepted with a data rate of $48 \mathrm{kbps}$. Video calls with high resolution are accepted with a data rate of $384 \mathrm{kbps}$. Data calls are accepted with a minimum data rate of $64 \mathrm{kbps}$ and it can achieve a maximum data rate of $384 \mathrm{kbps}$. It is assumed that the load threshold factor is 0.8, WCDMA chip rate is $3.84 \mathrm{Mcps}$, the orthogonality factor is 0.5 and the other cell to own cell interference ratio factor is 0.65 .

\begin{tabular}{|c|c|c|c|c|c|}
\hline Parameter & Voice Call & & $\begin{array}{l}\text { Data } \\
\text { Call }\end{array}$ & $\begin{array}{l}\text { Low } \\
\text { Resolution } \\
\text { Video Call } \\
\end{array}$ & $\begin{array}{l}\text { High } \\
\text { Resolution } \\
\text { Video Call }\end{array}$ \\
\hline $\begin{array}{l}\text { WCDMA } \\
\text { chip rate }\end{array}$ & \multicolumn{5}{|l|}{ 3.84 Mcps } \\
\hline Orthogonality & \multicolumn{5}{|l|}{0.5} \\
\hline $\begin{array}{l}\text { Other Cell to } \\
\text { Own Cell } \\
\text { Interference } \\
\text { Ratio }\end{array}$ & \multicolumn{5}{|l|}{0.65} \\
\hline $\begin{array}{l}\text { Load } \\
\text { Threshold }\end{array}$ & \multicolumn{5}{|l|}{0.8} \\
\hline \multirow{2}{*}{$\begin{array}{l}\text { Activity } \\
\text { Factor }\end{array}$} & Uplink & 0.67 & \multirow{2}{*}{1} & \multirow{2}{*}{1} & \multirow{2}{*}{1} \\
\hline & Downlink & 0.58 & & & \\
\hline $\begin{array}{lr}\text { Signal } & \text { to } \\
\text { Noise } & \text { Ratio } \\
\left(\mathrm{E}_{\mathrm{b}} / \mathrm{N}_{\mathrm{o}}\right) & \\
\end{array}$ & $7.0 \mathrm{db}$ & & $\begin{array}{l}5.0 \\
\mathrm{db}\end{array}$ & $4.0 \mathrm{db}$ & $3.2 \mathrm{db}$ \\
\hline Data Rate & $12.2 \mathrm{kbps}$ & & $\begin{array}{l}64 \\
\text { kbps }\end{array}$ & $48 \mathrm{kbps}$ & 384 kbps \\
\hline
\end{tabular}
A list of other parameters and assumptions for the UTRAN network are shown in Table I [19].

TABLE I. UTRAN PARAMETERS

The load factors for uplink and downlink in UTRAN are calculated as follows [19]:

$$
\eta_{U L}=(1+i) ? \sum_{j=1}^{N} \frac{1}{1+\frac{W}{\left(E_{b} / N_{o}\right)_{j} ? R_{j} \quad v_{j}}}
$$


where $\mathrm{N}$ is the number of users per cell, $v_{\mathrm{j}}$ is the activity factor of user $j$ at physical layer, $\mathrm{E}_{\mathrm{b}} / \mathrm{N}_{\mathrm{o}}$ is the signal energy per bit divided by noise spectral density, $\mathrm{W}$ is the chip rate, $R_{j}$ is the data rate of user $j$ and $i$ is the other cell to own cell interference ratio seen by the base station receiver. The load factor for the downlink is:

$$
\eta_{D L}=\sum_{j=1}^{N} v_{j} \times \frac{\left(E_{b} / N_{o}\right)_{j}}{W / R_{j}} \times\left[\left(1-\alpha_{j}\right)+i_{j}\right]
$$

where $\alpha_{j}$ is the orthogonality of channel of user $j$ and $i_{j}$ is the ratio of other cell to own cell base station received by user $j$.

In LTE, the transmission scheme is based on Orthogonal Frequency Division Multiple Access (OFDMA) for downlink and Single Carrier Frequency Division Multiple Access (SC-FDMA) for uplink. The allocated bandwidth is divided into a number of physical Resource Blocks (RBs) in term of frequency, each one has $180 \mathrm{KHz}$. Each RB is divided into 84 Resource Elements (12 subcarriers in terms of frequency $\times 7$ symbols in terms of time), each one has a $15 \mathrm{KHz}$ bandwidth. The load factor for connected user $j$ is calculated as follows:

$$
L_{j}=\frac{R B_{j} \times\left(1+R_{j}\right) \times v_{j}}{f r_{j}}
$$

where

$\mathrm{RB}_{\mathrm{j}}$ is the required number of resource blocks to transmit in one Transmission Time Interval (TTI) for user $j$.

$\mathrm{R}_{\mathrm{j}}$ is the average ratio of retransmission for user $j$.

$v_{\mathrm{j}}$ is the activity factor of user $j$.

$f r{ }_{j}$ is the average TTI where a new packet is transmitted from user $j$.

In WLAN, the transmission scheme is also based on OFDMA. Load of WLAN is calculated by the following equation:

$$
L_{W}=\sum_{j=1}^{N} \frac{R_{j} \times v_{j}}{W_{C}}
$$

where

$\mathrm{N}$ is the number users served in WLAN,

$R_{j}$ is the data rate of user $j$,

$v_{\mathrm{j}}$ is the activity factor of user $j$,

$\mathrm{W}_{\mathrm{C}}$ is the available WLAN capacity.

\section{PERFORMANCE RESUlTS}

The proposed intelligent mobility optimization and intelligent cheapest cost algorithms are compared with the centralized load-balancing, and the distributed algorithms in terms of new call blocking probability, VHO call dropping probability, users' satisfactions probability, average satisfaction increasing percentages, load utilization, average cell throughput, average cost per call and average saved cost percentage per call.

The centralized load-balancing algorithm aims to distribute traffic load between all available RATs in a heterogeneous wireless network. Balancing load between all available RATs in a heterogeneous wireless network offers an efficient utilization of the radio resources [2022]. In the centralized load-balancing algorithm, calls are allocated to the RAT that has the minimum load which is based on the decision made by the core network or the base station.

Two distributed algorithms are considered: distributed mobility and distributed cheapest cost algorithms. In the distributed mobility algorithm, the RAT selection decision is made by the UT, where the users with high mobility select the RATs that have the highest geographical coverage without considering any of the network factors such as network capacity and supported service type for the selected RAT. In the distributed cheapest cost algorithm, the RAT selection decision is made by the UT, where calls are allocated to the RAT that offers the cheapest cost for the user without considering any of the network factors for the selected RAT.

The proposed intelligent mobility optimization and intelligent cheapest cost algorithms aim to increase users' satisfactions by allocating users with high mobility to a RAT that has high coverage area and users that are looking for cheapest cost connection to a RAT that offers the cheapest cost of service and at the same time, they aim to improve the efficiently of radio resource utilization by minimizing unnecessary handover. More details on these algorithms are available in Section II.

Fig. 4 shows the blocking probability for the five RAT selection algorithms. Blocking probabilities are calculated as follows:

$$
P_{\text {bloc }}=\frac{N_{\text {bloc }}}{N_{\text {New }}}
$$

where

$\mathrm{N}_{\text {bloc }}$ is the number of blocked new calls.

$\mathrm{N}_{\text {New }}$ is the total number of requested new calls.

This figure shows that the proposed intelligent mobility optimization and intelligent cheapest cost algorithms outperform the centralized load-balancing and the distributed algorithms in term of blocking probability. The proposed algorithms perform better than the centralized load-balancing algorithm because the loadbalancing algorithm leads to high levels of unnecessary VHO which reduces the overall network capacity. In addition, the network capacity is reduced in centralized load-balancing algorithm as a result of the signaling load and delays introduced by the communication between the network entities during the RAT decision process. Distributed algorithms show the lowest levels of performance when compared to the other strategies. This is because the distributed algorithms do not consider any of the network factors such as load threshold. 


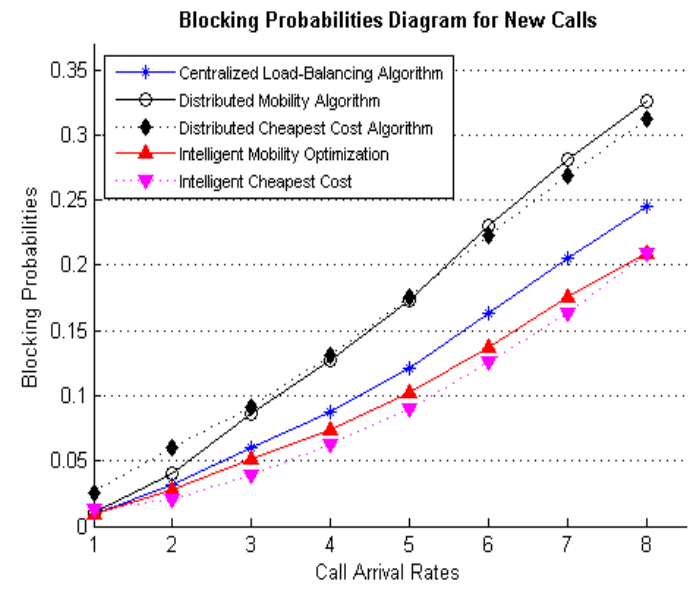

Figure 4. New calls blocking probability

Fig. 5 shows the dropping probability for the five RAT selection algorithms. Dropping probabilities are calculated as follows:

$$
P_{\text {drop }}=\frac{N_{\text {drop }}}{N_{V H O}}
$$

where

$\mathrm{N}_{\text {drop }}$ is the number of dropped VHO calls.

$\mathrm{N}_{\mathrm{VHO}}$ is the total number of requested VHO calls.

It can be seen that the proposed algorithms perform better than the centralized load-balancing algorithm as a result of the reduced network capacity introduced by the high unnecessary VHO and the increased signaling load produced during the communication between the network entities in the RAT decision process. The proposed algorithms outperform also the distributed algorithms which are the worst ones in term of dropping probability.

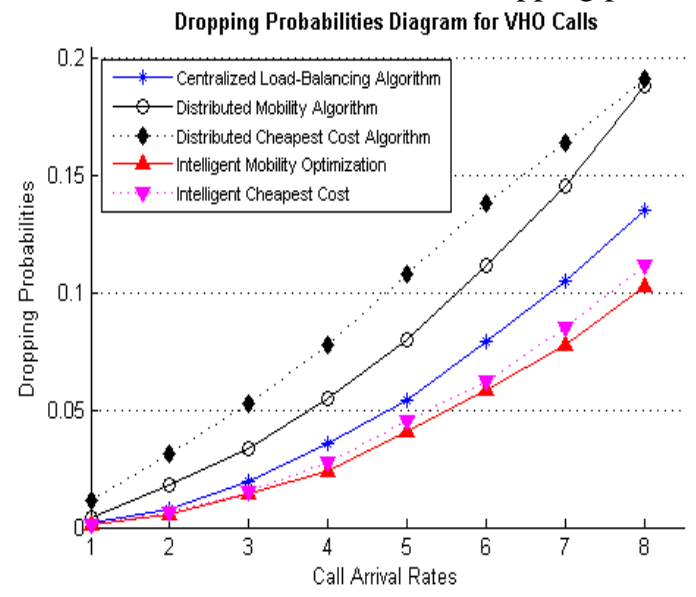

Figure 5. VHO calls dropping probability

Fig. 6 illustrates the users' satisfactions probability for the five RAT selection algorithms. The users' satisfactions probability is calculated by the following equation:

$$
P_{S}=\frac{N_{S_{-} \mathrm{New}}+N_{S_{-} \mathrm{VHO}}}{N_{\text {New }}+N_{V H O}}
$$

where
$\mathrm{N}_{\mathrm{S}_{-} \text {New }}$ is the number of satisfied new call users.

$\mathrm{N}_{\mathrm{S} \_ \text {VHO }}$ is the number of satisfied VHO call users.

In the intelligent and distributed mobility optimization algorithms, users are indicated as satisfied users if the users are in high mobility and have been allocated to the RAT that offers the highest coverage area between the available RATs when requesting new or VHO calls.

In the intelligent and distributed cheapest cost algorithms, users are indicated as satisfied users if they are looking for cheapest cost connections and have been allocated to the RAT that offers the cheapest cost of service between all available RATs when requesting new or VHO calls.

Simulation results show that the proposed and the distributed algorithms perform better than the centralized load-balancing algorithm. This is because, both proposed and distributed algorithms consider users' preferences which increase their satisfactions. Distributed and proposed algorithms have similar performance when traffic level is low. However, when the traffic becomes high, the proposed algorithms outperform the distributed algorithms in term of users' satisfactions probability. This is because the distributed algorithms do not consider any of the network factors such as load threshold.

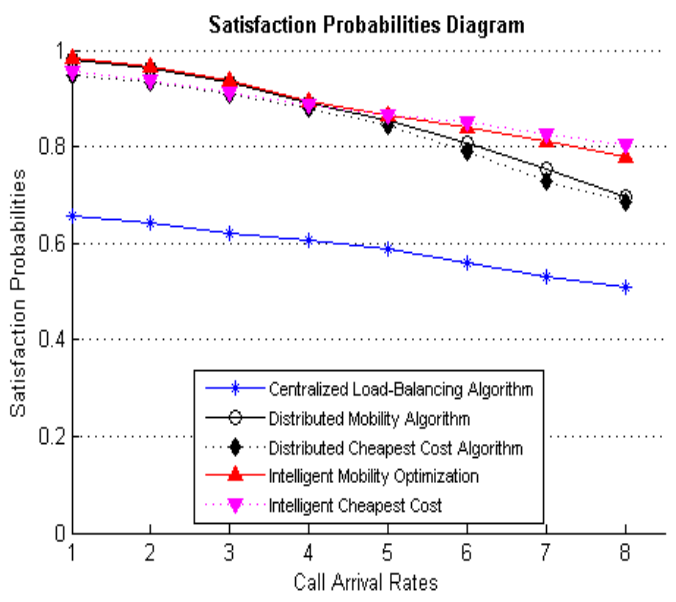

Figure 6. Users' satisfactions probability

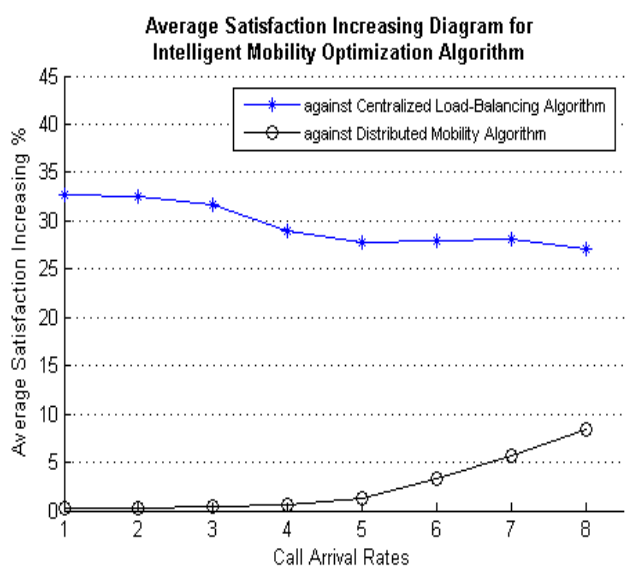

Figure 7. Average satisfaction increasing percentages for the proposed intelligent mobility optimization algorithm.

Fig. 7 shows the average satisfaction increasing percentages for the proposed intelligent mobility optimization algorithm compared with the centralized 
load-balancing and the distributed mobility algorithms. Average satisfaction increasing percentages are calculated by subtracting the users' satisfactions probability of the load-balancing and distributed algorithms from the users' satisfactions probability of the proposed algorithm. This figure shows that the users' satisfactions have been increased around 33\% against the centralized algorithm when traffic load is low and around $27 \%$ when traffic load is high. It also shows that the users' satisfactions percentages for the proposed algorithm are similar to the distributed algorithm when traffic load is low; however, users' satisfactions have been increased around $9 \%$ when traffic load is high.

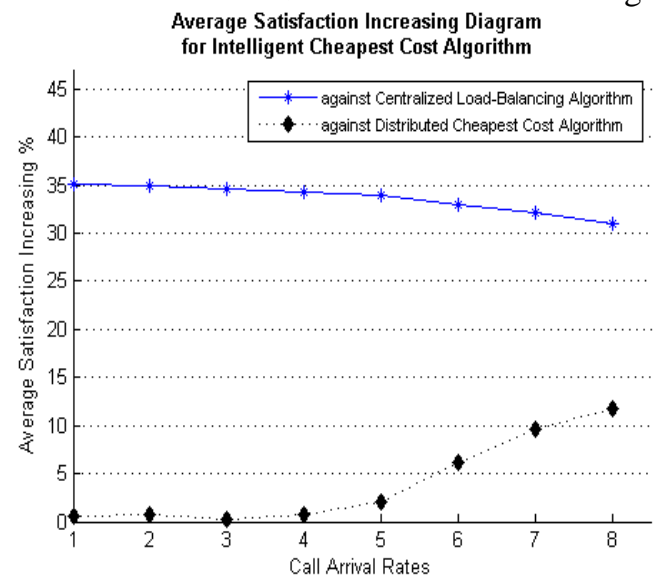

Figure 8. Average satisfaction increasing percentages for the proposed intelligent cheapest cost algorithm.

Fig. 8 shows the average satisfaction increasing percentages for the proposed intelligent cheapest cost algorithm compared with the centralized load-balancing and the distributed cheapest cost algorithms. The figure shows that the satisfaction probability of the proposed cheapest cost algorithm is higher and has been increased around $33 \%$ against the centralized algorithm. The satisfaction probabilities of the proposed and distributed algorithms have similar performance when traffic load is low; however, the satisfaction probability of the proposed algorithm has been increased up to $12 \%$ when traffic load becomes high.

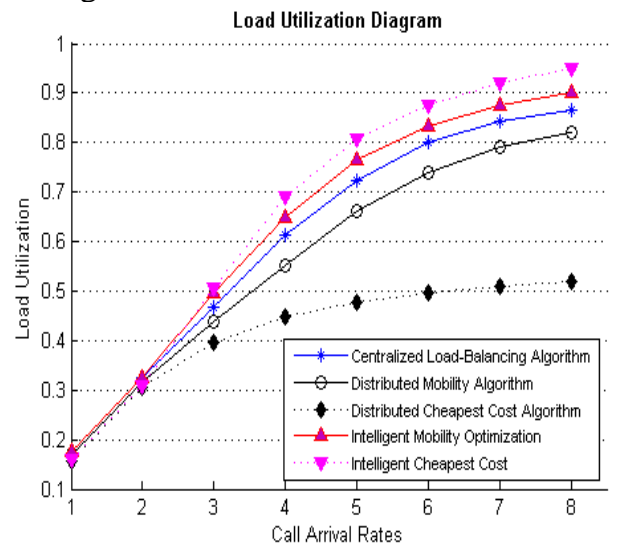

Figure 9. Load utilization

Fig. 9 and Fig. 10 show the load utilization and the average cell throughput for the five RAT selection algorithms. These figures show that all algorithms have similar performance when traffic level is low. However, when the traffic level becomes high, the proposed algorithms outperform the distributed and the centralized algorithms. This is because, in the distributed algorithm, users do not have the required information about cell load of each RAT which leads to keep the capacity of some RATs unused. In the centralized algorithm, the capacity is reduced as a result of increased signalling load and unnecessary VHO.

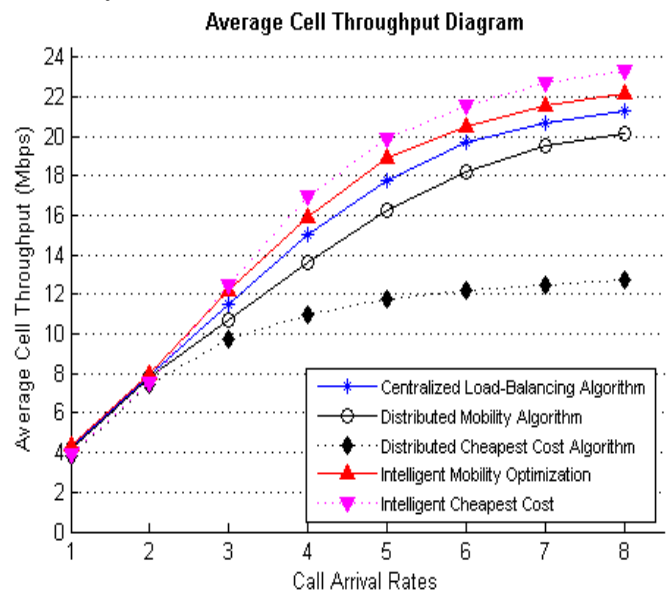

Figure 10. Average cell throughput

Fig. 11 shows the average cost per call of UTs that prefer to be allocated to the cheapest RAT cost for the centralized, intelligent cheapest cost and distributed cheapest cost algorithms. Simulation results show that the intelligent and distributed cheapest cost algorithms perform better than the centralized algorithm. This is because the centralized algorithm does not consider users' preferences. The distributed cheapest cost algorithm allocates calls to the RAT that offers the lowest cost to the UT device. The intelligent cheapest cost algorithm considers users' preferences and at the same time balances these preferences and the network factors such as supported service type and load threshold. The intelligent and the distributed algorithms have similar performance in terms of average cost per call.

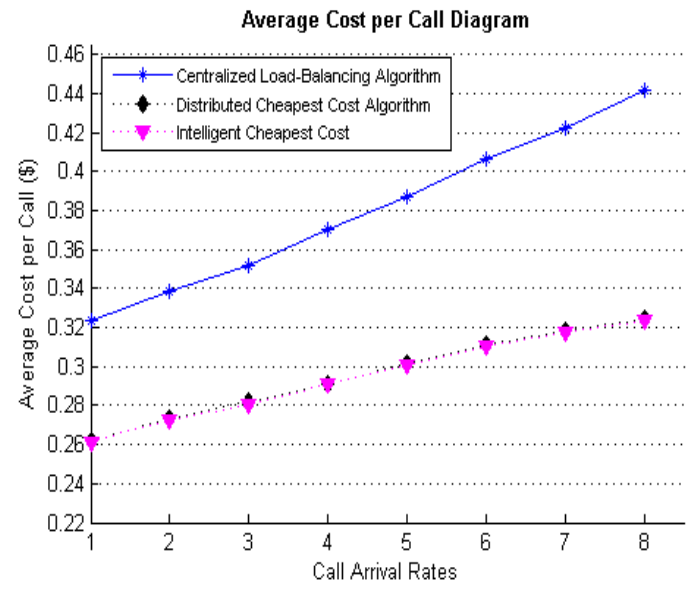

Figure 11. Average cost per call

Fig. 12 shows the average saved cost percentage per call for UTs that prefer to be allocated to the cheapest 
RAT cost for the proposed intelligent cheapest cost algorithm compared with the centralized algorithm. This figure shows that users which prefer to be allocated to the cheapest RAT cost can save between $23.8 \%$ and $36.6 \%$ per call in the proposed cheapest cost algorithm compared to the centralized RAT selection algorithm.

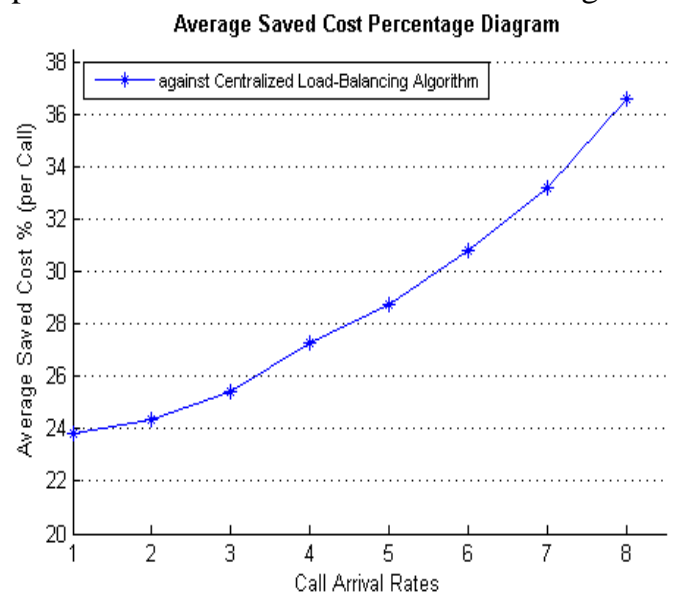

Figure 12. Average saved cost percentage per call

\section{CONCLUSIONS}

An intelligent RAT selection approach is necessary for a more efficient utilization of radio resources in heterogeneous wireless networks and to increase users' satisfactions and guarantee the required QoS for allocated calls. This paper presents the intelligent RAT selection approach for mobility optimization and proposes an intelligent cheapest cost RAT selection approach for heterogeneous wireless networks. The proposed algorithms have been compared with the centralized loadbalancing and the distributed algorithms in terms of new call blocking probability, VHO call dropping probability, users' satisfactions probability, average satisfaction increasing percentages, load utilization, average cell throughput, average cost per call and average saved cost percentage per call. Simulation results show that the proposed algorithms are more efficient than the centralized and the distributed algorithms.

\section{ACKNOWLEDGMENT}

This work is sponsored by the Centre for Real-Time Information Networks (CRIN) in the Faculty of Engineering \& Information Technology at the University of Technology, Sydney (UTS).

\section{REFERENCES}

[1] Motorola, "Technical White Paper: Long Term Evolution (LTE): A Technical Overview," 2010.

[2] V. K. Garg, Wireless Communications and Networking, Morgan Kaufmann Publishers, San Francisco, CA, USA 2007.

[3] P. Nicopolitidis, M. S. Obaidat, G. I. Papadimitriou, and A. S. Pomportsis, Wireless Networks, John Wiley \& Sons Ltd, Chichester, UK 2003.

[4] J. Schiller, Mobile Communications, 2nd Edition, Addison Wesley, Harlow UK 2003.
[5] A. AL Sabbagh, R. Braun and M. Abolhasan, "Centralized and Distributed CRRM in Heterogeneous Wireless Networks", in Advanced Methods and Applications in Computational Intelligence, Topics in Intelligent Engineering and Informatics, vol. 6, Springer International Publishing, Switzerland 2014, pp. 299-314.

[6] O. E. Falowo, "Terminal-Modality-Based Joint Call Admission Control Algorithm for Fair Radio Resource Allocation in Heterogeneous Cellular Networks," International Journal of Communications, Network and System Sciences, Vol. 5, no. 7, July 2012, pp. 392-404.

[7] G. Koundourakis, D. I. Axiotis and M. Theologou, "Network-Based Access Selection in Composite Radio Environments," IEEE Wireless Communications and Networking Conference (WCNC 2007), Hong Kong, March 11-15, 2007, pp. 3880-3886.

[8] D. Qiang, X. Dong-Liang, H. Bo, S. Yan and C. Shan-Zhi, "Joint Admission Control through Vertical Handoffs in Heterogeneous Wireless Networks," The 2010 Global Mobile Congress (GMC 2010), Shanghai, China, October 18-19, 2010, pp. 1-5.

[9] A. AL Sabbagh, R. Braun and M. Abolhasan, "A Comprehensive Survey on RAT Selection Algorithms for Heterogeneous Networks," World Academy of Science, Engineering and Technology (WASET), no. 73, January 2011, pp. 141-145.

[10] A. AL Sabbagh, R. Braun and M. Abolhasan, "A Comprehensive Survey on RAT Selection Algorithms for Beyond 3G Networks," in International Conference on Communications, Networking and Mobile Computing (ICCNMC 2011), Dubai, UAE, January 25-27, 2011, pp. 834-838.

[11] E. Adamopoulou, K. Demestichas, A. Koutsorodi and M. Theologou, "Intelligent Access Network Selection in Heterogeneous Networks," in 2nd International Symposium on Wireless Communication Systems 2005 (ISWCS'05), Siena, Italy, September 5-7, 2005, pp. 279283.

[12] F. Bari and V. C. M. Leung, "Automated Network Selection in a Heterogeneous Wireless Network Environment," IEEE Network, vol. 21, no. 1, January February 2007, pp. 34-40.

[13] Q. T. Nguyen-Vuong, N. Agoulmine and Y. GhamriDoudane, "Terminal-Controlled Mobility Management in Heterogeneous Wireless Networks," IEEE Communications Magazine, vol. 45, no. 4, April 2007, pp. 122-129.

[14] A. AL Sabbagh, R. Braun and M. Abolhasan, “A Mobility Optimization CRRM Approach for Next Generation Wireless Networks," International Conference on Computer and Information Sciences (ICCIS 2012), Kuala Lumpur, Malaysia, June 12-14, 2012, pp. 609-613.

[15] A. AL Sabbagh, R. Braun and M. Abolhasan, "A Power Efficient RAT Selection Algorithm for Heterogeneous Wireless Networks," in 2012 International Symposium on Communications and Information Technologies (ISCIT 2012), Gold Coast, Australia, October 2-5, 2012, pp. 997 1002.

[16] A. AL Sabbagh, "A Hybrid Radio Resource Allocation Method for Multiple Wireless Access Networks," Australian Innovation Patent AU2013100801, July 2013.

[17] S. Buljore, et al., "IEEE P1900.4 System Overview on Architecture and Enablers for Optimised Radio and Spectrum resource usage," in 2008 IEEE Symposium on New Frontiers in Dynamic Spectrum Access Networks, Chicago, Illinois, USA, October 14-17, 2008. 
[18] ETSI Technical Report, "Universal Mobile Telecommunications System (UMTS), Selection procedures for the choice of radio transmission technologies of the UMTS," UMTS 30.03 version 3.2.0, March 1998.

[19] H. Holma and A. Toskala, WCDMA for UMTS - HSPA Evolution and LTE, fourth Edition, John Wiley \& Sons Ltd, Chichester, UK 2007.

[20] A. Tolli and P. Hakalin, "Adaptive Load Balancing Between Multiple Cell Layers," in 2002 IEEE 56th Vehicular Technology Conference (VTC 2002), vol. 3, Vancouver, Canada, September 24-28, 2002, pp. 16911695.

[21] K. H. Suleiman, H. A. Chan and M. E. Dlodlo, "Load Balancing in the Call Admission Control of Heterogeneous Wireless Networks," in International Conference on Communications and Mobile Computing (IWCMC 2006), Vancouver, British Columbia, Canada, July 3-6, 2006, pp. 245-250.

[22] A. Pillekeit, F. Derakhshan, E. Jugl and A. Mitschele-Thiel, "Force-Based Load Balancing in Co-located UMTS/GSM Networks," in 2004 IEEE 60th Vehicular Technology Conference (VTC 2004), vol. 6, Los Angeles, California, USA, September 26-29, 2004, pp. 4402-4406.

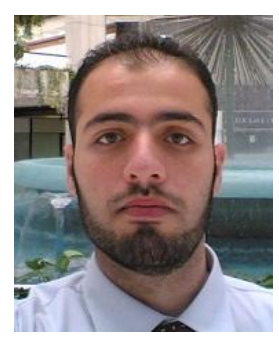

Abdallah AL Sabbagh received his B.Sc. in Information Technology and Computing (Hons) from the Arab Open University, Lebanon and the Open University, UK (2006) and a Master of Engineering Studies (MES) in Telecommunication Networks from the Faculty of Engineering at the University of Technology, Sydney (2010). He is currently with the School of Computing and Communications, and Centre for Real-Time Information Networks (CRIN) at the University of Technology, Sydney (UTS). His recent research is the Radio Resource Management (RRM), Admission Control, Vertical Handover (VHO) and packet scheduling for Next Generation Wireless Networks (NGWN). Abdallah is member of the Centre for Real-time Information Networks (CRIN) and Engineers Australia.

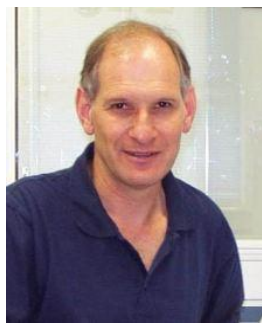

Robin Braun received his B.Sc (Hons) from Brighton University (UK), and his M.Sc and Ph.D from the University of Cape Town. He holds the Chair of Telecommunications Engineering in the Faculty of Engineering and Information Technology of the University of Technology, Sydney, Australia. He is an executive member of the Centre for Real Time Information Networks (CRIN) at the University of Technology, Sydney (UTS). Prof. Braun was a member of staff of the Department of Electrical Engineering of the University of Cape Town from 1986 to 1998. He was the founder, and Director of the Digital Radio Research Group at the University of Cape Town, which supervised over 50 research degree candidates in the years that he was attached to it. Prof. Braun is currently a Senior Member of the Institute of Electrical and Electronic Engineers of the United States (IEEE).

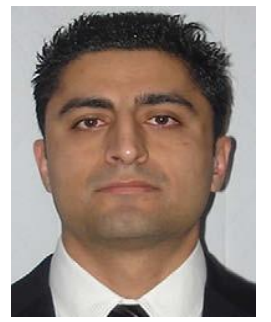

Mehran Abolhasan completed his B.E in Computer Engineering and Ph.D. in Telecommunications on 1999 and 2003 respectively at the University of Wollongong. From July 2003, he joined the Smart Internet Technology CRC and Office of Information and Communication Technology within the Department of Commerce in NSW, where he proposed a major status report outlining strategies and new projects to improve the communications infrastructure between the NSW Emergency Services Organisations. In July 2004, he joined the Desert knowledge CRC and Telecommunication and IT Research Institute to work on a joint project called the Sparse Ad hoc network for Deserts project (Also known as the SAND project). Dr. Abolhasan is currently a Senior Lecturer at the School of Computing and Communications within the faculty of Engineering and IT (FEIT) at the University of Technology Sydney (UTS). He has authored over 80 international publications and has won over one million dollars in research funding over the past 5 years. His Current research Interests are in Wireless Mesh, 4th Generation Cooperative Networks and Sensor networks. Dr. Abolhasan is currently a Senior Member of the Institute of Electrical and Electronic Engineers of the United States (IEEE). 